\title{
Answer to the Letter to the Editor of L. Denteneer et al. concerning "Do we have the right PROMs for measuring outcomes in lumbar spinal surgery?"' by O.M. Stokes et al., Eur Spine J (2017) 26:816-824
}

\author{
Oliver M. Stokes ${ }^{1}$
}

Received: 20 September 2017 / Accepted: 23 September 2017 / Published online: 3 November 2017

(C) Springer-Verlag GmbH Germany 2017

I would like to thank the readers for their interest in our study and for taking the time to make insightful comments in their Letter to the Editor. Our review of PROMs in lumbar spinal surgery was designed to identify the main outcome measures that are used to assess outcomes in spinal surgery patients and to determine if the measures currently used are accurate and reliable. The initial search identified more than 30 PROMs. The most commonly reported generic and condition-specific outcome measures were described and evaluated.

Modifications to the Oswestry Disability Index (ODI) are described in our study, but not in detail. I thank the reader for their letter, which provides greater information on the background to and the impact of the modifications to ODI. This does not, however, change our conclusion that more work is needed to improve PROMs.
I agree that the modified version of ODI is often more applicable to our patients and that missing data, when some patients do not complete entire PROMs, can influence the results. The readers are correct that our search of the literature did not differentiate between the different versions of the ODI. I would like to thank the readers for highlighting the important distinction between these outcome measures and for their efforts to evaluate the MDQ.

Compliance with ethical standards

Conflict of interest None of the authors have any potential conflicts of interest.
Oliver M. Stokes

oliverstokes@hotmail.com

1 Exeter Spine Unit, Princess Elizabeth Orthopaedic Centre, Royal Devon and Exeter NHS Foundation Trust, Exeter, UK 